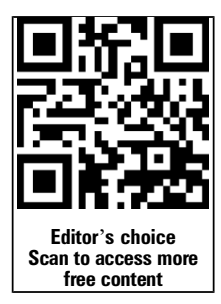

For numbered affiliations see end of article.

\section{Correspondence to} Dr D Rowley, Sydney Sexual Health Centre, Sydney Hospital, Macquarie St, NSW 2000, Australia;

dominicrowley@hotmail.com

Received 4 September 2014 Revised 23 January 2015 Accepted 1 February 2015 Published Online First 5 March 2015
CrossMark

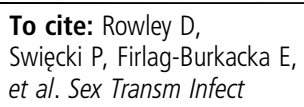

\title{
Clinical and epidemiological characteristics of patients with early syphilis from three academic centres in Poland, Germany and Ireland: initial findings from the POETS study
}

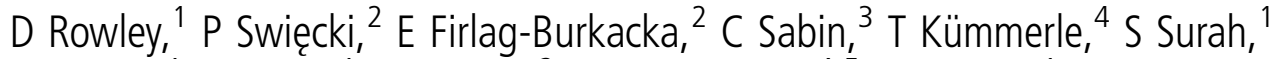 \\ C Sadlier, ${ }^{1}$ S O'Dea, ${ }^{1}$ A Horban, ${ }^{2}$ G Fätkenheuer, ${ }^{4,5}$ F Mulcahy $^{1}$
}

\section{ABSTRACT}

Objectives Syphilis recognition in HIV-positive patients has important implications. Initial data from this study, established in June 2012 to better understand the natural history of syphilis and treatment response, examine the characteristics of patients including sexual behaviour, rates of concurrent sexually transmitted infections (STI) and type of treatment given.

Methods Patients were recruited from Ireland, Poland and Germany. Data gathered included demographics, method of syphilis acquisition, stage of syphilis infection, HIV status, nadir and current CD4 counts and HIV viral suppression rates. Data were then subanalysed into HIV-positive and HIV-negative groups.

Results of 175 patients recruited, $68 \%$ were HIV-positive and $86.3 \%$ were men who have sex with men. Most HIV-positive patients presented with secondary syphilis ( $55.7 \%$ vs $13.2 \%$ ) ( $p=0.0001$ ) while the majority of HIV-negative patients had primary syphilis noted at the time of recruitment ( $47.2 \%$ vs $18.9 \%$, $\mathrm{p}=0.0002$ ). Approximately half of all patients had a HIV RNA viral load $<40$ copies $/ \mathrm{mL}$ (55\%). Previous syphilis infection occurred more frequently in HIV-positive than HIV-negative patients ( $p=0.0001$ ). Concurrent STls at the time of syphilis diagnosis were found in $26.8 \%$, of whom 31 (25.4\%) were HIV-positive ( $p=0.64)$. HIVpositive patients received doxycycline more frequently than their HIV-negative counterparts (33.6\% vs $1.9 \%$, $\mathrm{p}=0.0001$ ) while HIV-negative patients were treated with long-acting penicillin in $88.7 \%$ of cases vs $58 \%$ of HIV-positive patients ( $p=0.0002$ ).

Conclusions A $40 \%$ rate of unsuppressed viraemia, high levels of STIs and varying treatment regimens represent a public health risk for Europe, suggesting the model of sexual healthcare delivery in HIV-positive patients requires further evaluation.

\section{INTRODUCTION}

Syphilis is a sexually transmitted infection (STI) caused by the spirochete Treponema pallidum. It has a long and rich history with cases of treponemal infection being documented in pre-Columbus Europe, Africa and Asia. ${ }^{1}$

Following the Second World War, with increased access to penicillin and better public health policies addressing venereal diseases, the rates of syphilis infection dropped substantially in high-income settings. ${ }^{2}$ However, since the turn of the 21 st century, the incidence of syphilis has risen globally. ${ }^{3-6}$ Ireland, Poland and Germany have also followed this trend with reported cases of syphilis in 2012 of 518, 963 and 4410, respectively, with the majority occurring among men who have sex with men (MSMs)..$^{3-5} 7$

This increased incidence is a result of widely differing underlying factors that include economic, social and technological factors, ${ }^{8}$ along with migratory and stigma-related issues. ${ }^{9}{ }^{10}$ The rise has occurred most dramatically among MSMs, and syphilis now disproportionately affects people who are HIV-positive, particularly HIV-positive MSMs. ${ }^{11-14}$ The reasons for this are complex and manifold; the increased incidence cannot simply be explained by a rise in HIV infections alone since many countries with rising rates of syphilis infections have not seen a corresponding rise in HIV incidence. ${ }^{15}$ Changes in social norms and sexual behaviours including a greater rate of high-risk sex with a high turnover of sex partners, use of the internet to meet sex partners, serosorting (choosing a partner based on their HIV status), the use of recreational drugs, ${ }^{16}$ to heighten sexual experiences (eg, crystal methamphetamine), and a perception that oral sex is the safest option to prevent HIV acquisition ${ }^{17}$ have all likely contributed to the epidemic.

Early reports in the literature referred to HIV affecting the natural history of syphilis, but that is no longer thought to be the case. Some differences remain, however, between syphilis when it presents in the HIV-positive population compared with the HIV-negative population. Higher rates of asymptomatic primary infection, greater rates of chancres occurring in the secondary stage and overall much higher rates of patients presenting with secondary syphilis have all been documented. ${ }^{16}{ }^{18-20}$ There may also be greater numbers of chancres that are deeper in appearance and, finally, individuals with syphilis may present with or progress to neurological signs and symptoms more frequently. ${ }^{20}$

Syphilis facilitates the transmission and acquisition of HIV in a number of ways. At the time of primary infection, mucosal breaks in the skin due to anogenital ulceration cause loss of the skin's natural defence mechanism. ${ }^{19}$ Within these ulcers, syphilitic-infected macrophages undergo CCR5 
receptor expression as a result of syphilitic surface lipoprotein stimulation. These CCR5-induced cells then act as a reservoir for macrotrophic CCR5-positive HIV virions to attack. ${ }^{21}$ The systemic secondary stage is marked by a potent cellular response causing increased numbers of activated CD4+ cells to circulate, which likely increase the transmission of HIV. Syphilis also alters the natural history of the HIV infection as HIV viral load rises and CD4 cell counts drops at the time of infection. ${ }^{22} 23$

To date, there have been few prospective randomised clinical trials examining the treatment of syphilis particularly in HIV-positive persons. One study revealed that clinical treatment failures were rare among both HIV-positive and HIV-negative persons but that serological failures were more common in those with HIV infection. The authors of this study concluded, however, that treatment was adequate regardless of HIV status. ${ }^{24}$ A recent systematic review examining the treatment of syphilis in HIV-infected subjects found there was no known optimal antibiotic regimen for those who were coinfected with syphilis and HIV, ${ }^{25}$ with most sites choosing parenteral forms of penicillin, oral doxycycline, azithromycin or ceftriaxone.

Comprehensive guidelines exist in different jurisdictions for the management of syphilis, ${ }^{26-28}$ all of which make special reference to HIV coinfected patients in whom it is advised to treat as per HIV-negative patients.

The prospective observational European study of the natural history and treatment of syphilis in HIV-positive and HIV-negative individuals (POETS) study was primarily designed to assess the natural history of syphilis infection along with treatment response in HIV-positive versus HIV-negative persons. It is anticipated that this would inform future guidelines. In this paper, we provide a summary of the patients recruited to the study, and associations found between lifestyle, sexual behaviour and other clinical factors such as their HIV status, viral suppression rates and staging of syphilis infection.

\section{METHODS}

A prospective multicentre cohort study was conducted examining all patients newly diagnosed with syphilis infection attending three tertiary referral university hospitals in Ireland, Poland and Germany. Included individuals could be HIV-positive or HIV-negative.

In Ireland, patients were recruited from a combined genitourinary medicine and infectious disease clinic where a mix of HIV-positive and general STI patients attend. Ten doctors provided care for the Irish cohort.

In Germany and Poland, patients were recruited from an academic HIV/infectious disease outpatient clinic and treated by five providers, respectively.

Demographic data were gathered including information on age, sexual orientation, country of birth, HIV status, whether or not the individual had a partner, and if this partner was aware of the person's HIV or syphilis diagnosis. The type of sexual activity that had led to the individual's diagnosis of syphilis, whether or not they had visited a bar, nightclub or a sex on premises venue, used the services of a commercial sex worker or consumed alcohol and/or illicit drugs was ascertained. The number and type of past STIs and the presence of a concurrent STI was noted.

Syphilis was diagnosed using a combination of treponemal tests and non-treponemal tests.

Treponemal tests included fluorescent treponemal antibody absorbed, T. pallidum particle agglutination and immunoglobulin G to T. pallidum as detected by enzyme immunoassay.
Non-treponemal tests included the Venereal Disease Research Laboratory test or the rapid plasma regain test.

Dark field microscopy was used in conjunction with these tests in a number of cases of syphilis with chancres present.

Syphilis infection was staged according to primary, secondary, latent (early or late) and tertiary. The presence of chancres, rash, lymphadenopathy and any cardiac and neurological signs was recorded. In addition, any neurological symptoms or penicillin allergies were documented. For those with HIV infection, length of time since HIV diagnosis, nadir CD4 count, current CD4 count, viral load and highly active antiretroviral therapy regimen were detailed. Finally, syphilis therapy was recorded.

A full STI screen was performed at baseline as per standard of care for MSMs. This included rectal, pharyngeal and urethral combined nucleic acid amplification test for chlamydia and gonorrhoea. HIV, hepatitis A, B, C and syphilis serology were also taken.

A swab for syphilis PCR, macrolide resistance and genotyping was taken from any primary ulcer site for future analysis.

Blood was taken for syphilis PCR, macrolide resistance and genotyping for patients presenting with secondary syphilis for future analysis.

\section{Statistical methods}

Comparisons between study participants in the three different countries and between those who were HIV-positive and HIV-negative were performed using $\chi^{2}$ test for categorical variables or Mann-Whitney U test for continuous variables. All analyses were performed using SAS V.9.13, and a $\mathrm{p}$ value $<0.05$ was considered to be statistically significant.

\section{RESULTS}

To date, the study has recruited 175 patients, $122(69.7 \%)$ of whom were HIV-positive and $53(30.3 \%)$ of whom were HIV-negative. Almost all were men (170/175, 97.1\%), of whom $151(86.3 \%)$ were homosexual/bisexual and 22 (12.6\%) heterosexual. Although most patients reported being born in one of the participating countries (Ireland, Poland or Germany), 39 $(22.3 \%)$ reported other countries of birth (table 1). All were resident in the local country at the time of presentation.

The way in which patients met future sex partners varied between the three sites as did their alcohol and recreational drug consumption. The presence of a partner, along with the partner's awareness of the patient's HIV status and syphilis coinfection, also differed (table 1).

Among the HIV-positive patients, viral suppression rates ranged from $48.0 \%$ to $73.3 \%$. There was some degree of difference in terms of nadir CD4 count between Ireland, Poland and Germany, but the median CD4 counts at the time of syphilis diagnosis were well matched (table 2).

The 122 HIV-positive men in the study had a median age of 35 years compared with 31 years for the 53 HIV-negative patients $(\mathrm{p}=0.05)$.

Overall, $75(42.9 \%)$ individuals presented with secondary syphilis, $68(55.7 \%)$ of the HIV-positive group and 7 (13.2\%) of the HIV-negative group $(p=0.0001)$. Among those with secondary syphilis, a rash was present in 33 (48.5\%) of the HIV-positive group versus $4(57.1 \%)$ of the HIV-negative group $(\mathrm{p}=0.71)$.

Overall, $50(28.6 \%)$ individuals presented with primary syphilis, $23(18.9 \%)$ of the HIV-positive group and 27 (50.9\%) of the HIV-negative group $(p=0.0001)$. Among those with primary syphilis, a chancre was present in 12 (52.2\%) HIV-positive patients vs 15 (55.6\%) HIV-negative patients $(\mathrm{p}=1.002)$. Also, 8 (6.6\%) HIV-positive patients were classified 
Table 1 Demographic comparison between Ireland, Poland and Germany and differences across the three sites in the use of the internet, commercial sex workers, sex on premises venues and alcohol and recreational drugs

\begin{tabular}{|c|c|c|c|c|c|c|}
\hline & Ireland & Per cent & Poland & Per cent & Germany & Per cent \\
\hline Number of patients & 85 & 100.0 & 73 & 100.0 & 17 & 100.0 \\
\hline \multicolumn{7}{|l|}{ Gender } \\
\hline Male & 81 & 95.3 & 72 & 98.6 & 17 & 100.0 \\
\hline Female & 4 & 4.7 & 1 & 1.4 & 0 & 0 \\
\hline \multicolumn{7}{|l|}{ Age (years) } \\
\hline Median (range) & $35(19-77)$ & & $34(23-68)$ & & $35(21-56)$ & \\
\hline \multicolumn{7}{|l|}{ Country of birth } \\
\hline National & 49 & 57.7 & 72 & 98.6 & 15 & 88.2 \\
\hline Other European & 20 & 23.5 & 1 & 1.4 & 0 & 0 \\
\hline Africa & 3 & 3.6 & 0 & 0 & 0 & 0 \\
\hline South America & 10 & 11.8 & 0 & 0 & 0 & 0 \\
\hline North America & 1 & 0 & 0 & 0 & 0 & 0 \\
\hline Asia & 2 & 2.4 & 0 & 0 & 0 & 0 \\
\hline Middle East & 0 & 0 & 0 & 0 & 1 & 5.9 \\
\hline \multicolumn{7}{|l|}{ Sexual orientation } \\
\hline Homosexual/bisexual & 73 & 85.9 & 63 & 86.3 & 15 & 88.2 \\
\hline Heterosexual & 12 & 14.1 & 9 & 12.3 & 1 & 5.9 \\
\hline Not stated & 0 & 0 & 1 & 1.4 & 1 & 5.9 \\
\hline \multicolumn{7}{|l|}{ HIV status } \\
\hline Negative & 50 & 58.8 & 0 & 0 & 3 & 17.7 \\
\hline Positive & 35 & 41.2 & 73 & 100.0 & 14 & 82.4 \\
\hline Current partner & 34 & 40.0 & 8 & 11.0 & 7 & 50.0 \\
\hline Partner aware of Treponema pallidum & 21 & 61.8 & 1 & 12.5 & 8 & 57.1 \\
\hline Partner aware of $\mathrm{HIV}^{*}$ & 33 & 94.0 & 8 & 100.0 & 10 & 76.9 \\
\hline \multicolumn{7}{|l|}{ Mode of meeting partners } \\
\hline Internet & 42 & 49.4 & 0 & Not stated & 10 & 58.8 \\
\hline Clubs/bars & 48 & 56.5 & 64 & 87.7 & 3 & 17.7 \\
\hline Sex on premises venues (sauna, sex cinema) & 22 & 25.9 & 0 & 0 & 1 & 5.9 \\
\hline Commercial sex workers & 7 & 8.2 & 1 & 1.4 & 0 & 0 \\
\hline Alcohol use around time of sex & 59 & 69.4 & 62 & 84.9 & 7 & 41.2 \\
\hline \multicolumn{7}{|l|}{ Illicit oral/nasal 'party' drugs use } \\
\hline Ecstasy, ketamine, GBH'G', cocaine & 33 & 38.8 & 6 & 8.2 & 4 & 23.5 \\
\hline
\end{tabular}

${ }^{*}$ Refers to the partners of HIV-positive patients $(n=35)$.

as having early latent syphilis compared with $6(11.3 \%)$ HIV-negative patients $(\mathrm{p}=0.36)$ while $16(13.1 \%)$ HIV-positive patients had late latent disease compared with 9 (17.0\%) HIV-negative patients $(\mathrm{p}=0.66)$.

With regards to sexual behaviours and STIs, the HIV-positive group was found to be engaging in more anal-insertive and anal-receptive sex compared with their HIV-negative counterparts. There were also high rates of concurrent STIs in both groups with a significant proportion of HIV-positive patients being diagnosed with acute hepatitis $\mathrm{B}$ and $\mathrm{C}$ virus. A past history of syphilis infection was more common in the HIV-positive group (table 3).

Table 2 Viral suppression rates and CD4 counts in HIV-positive patients across three sites

\begin{tabular}{llll}
\hline HIV-positive & Ireland $(\mathbf{n}=\mathbf{3 5})$ & Poland $(\mathbf{n}=73)$ & Germany $(\mathbf{n}=\mathbf{1 4})$ \\
\hline $\begin{array}{l}\text { Virologically suppressed } \\
<39 \text { copies/mL }\end{array}$ & $22 / 30(73.3 \%)$ & $35 / 73(48.0 \%)$ & $7 / 12(58.3 \%)$ \\
$\begin{array}{l}\text { Current CD4 }\left(10^{6} / \mathrm{L}\right), \\
\text { median (range) }\end{array}$ & $570(64$ to 986$)$ & $460(6$ to 1214$)$ & $544(120$ to 1220$)$ \\
$\begin{array}{l}\text { Nadir CD4 }\left(10^{6} / \mathrm{L}\right), \\
\text { median (range) }\end{array}$ & $306(64$ to 907$)$ & $308(6$ to 1051$)$ & $200(15$ to 610$)$ \\
\hline
\end{tabular}

Within the HIV-positive group, 64 of the 115 patients with a viral load measurement $(55.7 \%)$ had a suppressed viral load (<39 copies $/ \mathrm{mL}$ ). Rates of reported oral sex, anal-insertive and anal-receptive sex and vaginal intercourse did not differ significantly between those with suppressed or non-suppressed viral loads (data not shown).

Treatment varied across the three sites $(p<0.0001)$; a longacting penicillin formulation was administered in 46.3\%, 19.4\% and $6 \%$ of cases in Ireland, Poland and Germany, respectively. Doxycycline was given in $1.7 \%, 21 \%$ and $1.7 \%$ of cases, ceftriaxone in $0 \%, 2.3 \%$ and $1.1 \%$ and azithromycin in $0 \%, 0.6 \%$ and $0.6 \%$ of cases, respectively.

In total, 71/122 (58\%) HIV-positive patients received a longacting penicillin formulation compared with 47/53 (88.7\%) HIV-negative patients $(p=0.0002)$. Forty-one $(33.6 \%)$ HIV-positive patients received doxycycline compared with only one $(1.9 \%)$ of the HIV-negative group $(p=0.0001)$. Two $(1.6 \%)$ HIV-positive patients were treated with azithromycin $(\mathrm{p}=1.0)$ while six $(4.9 \%)$ received ceftriaxone $(p=0.18)$.

\section{DISCUSSION}

Our study recruited broadly similar patient groups from all three sites in terms of age, sexual orientation and gender. Most were men, either homosexual or bisexual in orientation with a 
Table 3 Comparison of age, sexual orientation, sexual practices and past history of sexually transmitted infections (STIs) between HIV-positive and HIV-negative groups

\begin{tabular}{|c|c|c|c|}
\hline Patient & $\begin{array}{l}\text { HIV-negative } \\
(\mathrm{N}=53)\end{array}$ & $\begin{array}{l}\text { HIV-positive } \\
(\mathrm{N}=122)\end{array}$ & p Value \\
\hline Age (years), median (range) & 31 (19 to 62$)$ & 35 (19 to 77$)$ & 0.05 \\
\hline Homosexual & $36(67.9)$ & $96(78.7)$ & \\
\hline Bisexual & $8(15.1)$ & $11(9.0)$ & \\
\hline Heterosexual & $9(17.0)$ & $13(10.7)$ & \\
\hline Oral sex & $48(90.6)$ & $113(92.6)$ & 0.87 \\
\hline Anal-insertive sex & $30(56.6)$ & $95(77.9)$ & 0.007 \\
\hline Anal-receptive sex & $24(45.3)$ & $97(79.9)$ & 0.0001 \\
\hline Vaginal sex & $13(26.0)$ & $21(17.4)$ & 0.28 \\
\hline Fisting & $3(5.7)$ & $3(2.5)$ & 0.37 \\
\hline Concurrent STIs & $16(30.2)$ & $31(25.4)$ & 0.64 \\
\hline Anogenital warts & $4(7.6)$ & $8(6.6)$ & 0.76 \\
\hline Chlamydia & $7(13.2)$ & $7(5.7)$ & 0.13 \\
\hline Non-specific urethritis & $1(1.9)$ & $0(0)$ & 0.30 \\
\hline Gonorrhoea & $5(9.4)$ & $20(16.4)$ & 0.33 \\
\hline Herpes & $1(1.9)$ & $3(2.5)$ & 1.00 \\
\hline Acute hepatitis C & $0(0)$ & $7(5.7)$ & 0.10 \\
\hline Infectious hepatitis B & $1(1.9)$ & $8(6.6)$ & 0.28 \\
\hline $\begin{array}{l}\text { Acute hepatitis C+ } \\
\text { Infectious hepatitis B }\end{array}$ & $1(1.9)$ & $15(12.3)$ & 0.04 \\
\hline Past history of STIs & $20(37.7)$ & $64(52.2)$ & 0.1 \\
\hline Past syphilis infection & $4(7.6)$ & $54(44.3)$ & 0.0001 \\
\hline
\end{tabular}

median age of 34 years, with a wide variation in country of birth. This population is therefore reflective of a young, mobile population, acknowledged to be the group presently at the greatest risk of STI acquisition, particularly syphilis. ${ }^{2} 1114$

Social factors, including the way in which our patients met future sex partners, were examined with variations seen between the Western European and Eastern European sites. Alcohol use at the time of presumed syphilis acquisition was high with rates of $69.4 \%, 84.9 \%$ and $41.2 \%$ in Ireland, Poland and Germany, respectively, with illicit oral/nasal recreational drug use also reported frequently with rates of $38.8 \%, 8.2 \%$ and $23.5 \%$, respectively. We recognise that some of the differences between the sites are likely explained by response bias in terms of social desirability and stigma-related issues, but clearly social differences do exist between the three sites.

In terms of clinical presentation, only one in five of the HIV-positive patients had primary syphilis compared with almost half of the HIV-negative group $(\mathrm{p}=0.0002)$. We did not identify atypical syphilitic ulcers as noted in other clinical reports. ${ }^{18}$ This is in keeping with previous findings that primary syphilis may be symptomless in HIV-positive patients. ${ }^{20}$ Around half of the HIV-positive patients presented with secondary syphilis compared with only $13 \%$ of the HIV-negative patients, consistent with previous studies. ${ }^{16} 1929$

As expected, the rates of oral sex between the HIV-positive and HIV-negative groups were similar at $90.6 \%$ vs $92.6 \%$, although anal-insertive rates were higher among the HIV-positive patients $(77.9 \%$ vs $56.6 \%, \mathrm{p}=0.007)$ with anal-receptive rates of $79.9 \%$ and $45.3 \%(p=0.0001)$. When we further subdivided the group into those patients who had an HIV viral load $<39$ copies/mL and those with a viral load $>40$ copies $/ \mathrm{mL}$, we found no statistical differences between the rates of any types of sex, with high rates of all sexual activities (anal-insertive/anal-receptive and vaginal intercourse) across both groups. While it is of concern that there was a significantly greater amount of anal-insertive and anal-receptive sex among our HIV-positive patients, it is perhaps of greater concern that the virally non-suppressed patients did not appear to modify their sexual behaviour according to their viral load status. This has clear implications for healthcare providers in terms of delivering safe sex messages and risk reduction strategies to our HIV-positive patients. Interestingly, the levels of partner awareness regarding the recruited patients syphilis and HIV status indicated patients were more likely to have disclosed their HIV status rather than their syphilis infection to a current partner. This indicates that there may be a disconnect between the level of knowledge of increased transmissibility of HIV in the presence of another STI.

A high rate of concurrent STIs at the time of syphilis diagnosis was found in both HIV-positive and HIV-negative patients. Although no individual STI was found to be more common in the HIV-positive group, there was a significantly higher frequency of acute/infectious hepatitis C and B in those with HIV infection. A past history of STIs was noted in half of HIV-positive patients compared with $37 \%$ of HIV-negative patients, although it was noteworthy that a previous syphilis infection had occurred more frequently in the HIV-positive patients $(\mathrm{p}=0.0001)$.

Although much data exist ${ }^{30-32}$ regarding the treatment of syphilis, there are few studies that have compared the outcomes of treatment of HIV-positive and HIV-negative individuals, particularly with oral antibiotics compared with parenteral forms of penicillins. ${ }^{24}{ }^{33}$ A number of trials comparing the use of tetracyclines or macrolides versus long-acting penicillin have also shown to be equivalent, ${ }^{31} 32$ and, initially, azithromycin as a large single dose was thought to be a promising alternative. This was favoured due to the cumbersome and painful long-acting parenteral penicillins and clearance rates were good. ${ }^{30}$ However, the ever-growing epidemic of macrolide resistance around the world has prevented its recommended use. ${ }^{34-36}$ Interestingly, the most commonly referenced guidelines ${ }^{26} 2728$ all recommend parenteral penicillin as first-line treatment for HIV-positive and HIV-negative patients with syphilis; however, second-line and third-line therapy in HIV-positive patients remains contentious. Despite this, we found that only $58 \%$ of HIV-positive patients received a long-acting penicillin formulation compared with almost $90 \%$ of HIV-negative patients. In fact, the HIV-positive patients were more likely to receive oral doxycycline than the HIV-negative group. Reassuringly, only two HIV-positive patients received azithromycin while six received ceftriaxone. The administration of parental penicillin is dependent on the availability of on-site treatment clinic rooms and nursing staff, which may have been a factor favouring the use of oral tetracyclines in the context of an exclusive HIV service compared with a combined sexual health and HIV service.

The authors acknowledge a number of limitations that must be taken into consideration when analysing these data. First, as patients were recruited in a convenience sampling manner, with a relatively small German cohort, the findings may not be generalisable to all HIV-positive and HIV-negative MSMs in their respective countries. Second, the authors recognise that there may be bias in the results treatment administered due to a centre effect. Finally, this study was not designed to collect information about the probable route of transmission of infectious hepatitis $\mathrm{B}$ and $\mathrm{C}$ and partner serosorting, all of which may have provided useful information.

Despite these limitations, this prospective study recruited patients from both Eastern and Western Europe, the majority of whom were HIV-positive. The clinics chosen represent an accurate picture of where the majority of HIV-positive patients receive care in their respective countries and all three are 
specialist HIV sites. Follow-up treatment results in this study will undoubtedly inform future treatment guidelines of syphilis in HIV-positive patients.

European MSMs and, in particular, HIV-positive MSMs carry a disproportionate burden of STIs including syphilis infection and reinfection. There are a number of reasons for this, including social, economic and migratory, but what remains unclear is whether or not this increased incidence is reflective of an immunological susceptibility or whether this is due to increased treponemal exposure secondary to risky sexual behaviour. Good evidence exists that syphilis and HIV coinfection can affect a person's own disease trajectory (ie, viral load escapes and CD4 count drops) $)^{22} 23$ not to mention the risk of onward HIV transmission. Our study found a notable rate of concurrent STIs, $40 \%$ uncontrolled HIV vireamia and little apparent behaviour modification, regardless of viral load.

Targeted sexual healthcare in HIV-positive patients is vital, and the model of combined sexual health and HIV clinics needs further evaluation to optimise the assessment and management of these patients. We feel this has important implications for education and prevention strategies both for patients, their partners and their healthcare providers.

\section{Key messages}

- Syphilis infection continues to rise in Western and Eastern Europe, with men who have sex with men (MSMs), and in particular HIV-positive MSMs being disproportionately affected.

- This cohort of HIV-positive MSMs had significant high-risk sexual activity regardless of their viral load status and experienced high rates of concurrent sexually transmitted infections.

- HIV-positive MSMs were more likely to present with secondary syphilis and more likely to receive oral doxycycline than their HIV-negative counterparts.

- Treatment varied across all three sites despite the current guidelines on management of syphilis.

\author{
Author affiliations \\ ${ }^{1}$ The GUIDE (genitourinary and infectious disease) Clinic, St. James Hospital, Dublin, \\ Ireland \\ ${ }^{2}$ The Hospital for Infectious Diseases, Warsaw, Poland \\ ${ }^{3}$ University College London, Royal Free Campus, Rowland Hill street, London, \\ United Kingdom \\ ${ }^{4}$ Klinik I für Innere Medizin, Klinische Infektiologie, Uniklinik, Köln, Germany \\ ${ }^{5}$ German Centre for Infection Research (DZIF), Partner site Bonn-Cologne, Cologne, \\ Germany
}

Acknowledgements The authors specifically thank the patients for volunteering to participate in this study. The authors also thank Ellen Thomas at Klinik I für Innere Medizin, Klinische Infektiologie, Uniklinik Köln, Germany, for her help in data collection and patient recruitment.

Contributors FM and GF conceived the study. The original research protocol was written jointly by SOD and FM. DR, SOD, ET and PS were responsible for execution of the study. DR has written the manuscript, recruited the patients from Ireland and coordinated the three sites including the gathering together of all data. PS recruited patients in Poland and created the database. EFB recruited patients in Poland and maintained the database. CSab performed the statistical analysis and revised the manuscript. ET recruited patients in Germany and created and maintained the database. SS and CS recruited patients in Ireland. SOD created the database, recruited patients and maintained the data. AH was in charge of the study at the Infectious Disease Hospital in Warsaw. GF was in charge of the study in the Infectious Disease unit in Cologne. FM revised the manuscript, was the principal investigator and in charge of the study in Ireland. All authors had full access to the data in the study, and read, revised and approved the final manuscript.

\section{Competing interests None.}

Ethics approval Tallaght Hospital/St. James's Hospital Joint Research Ethics Committee, Dublin, Ireland. The ethical approval granted covered all three sites with additional ethical approval granted for the German cohort, allowing them to exchange pseudonymised data to multilateral projects.

Provenance and peer review Not commissioned; externally peer reviewed.

\section{REFERENCES}

1 Harrison LW. Origin of syphilis. Br J Vener Dis 1959;35:1-7.

2 Fenton $K A$, Breban $R$, Vardavas $R$, et al. Infectious syphilis in high-income settings in the 21st century. Lancet Infect Dis 2008:8:244-53.

3 Muldoon E, Mulcahy F. Syphilis resurgence in Dublin, Ireland. Int I STD AIDS 2011:22:493-7.

4 Majewski S, Rudnicka I. Sexually transmitted diseases in Poland in 2011. Przegl Epidemiol 2013:67:283-6, 379-81.

5 Bremer V, Marcus U, Hamouda O. Syphilis on the rise again in Germany-results from surveillance data for 2011. Euro Surveill 2012:17:pii:20222.

6 Bissessor M, Chen M. Syphilis, the great mimicker, is back. Aust Fam Physician 2009:38:384-7.

7 Health Protection Surveillance Centre. Syphilis in Ireland, 2012. 17 October 2013.

8 Nahmias SB, Nahmias D. Society, sex, and STIs: human behavior and the evolution of sexually transmitted diseases and their agents. Ann N Y Acad Sci 2011;1230:59-73

9 Zohrabyan L, Johnston L, Scutelniciuc O, et al. HIV, hepatitis and syphilis prevalence and correlates of condom use during anal sex among men who have sex with men in the Republic of Moldova. Int J STD AIDS 2013;24:357-64.

10 Xu JJ, Zhang M, Brown K, et al. Syphilis and HIV seroconversion among a 12-month prospective cohort of men who have sex with men in Shenyang, China. Sex Transm Dis 2010;37:432-9

11 Phipps W, Kent CK, Kohn R, et al. Risk factors for repeat syphilis in men who have sex with men, San Francisco. Sex Transm Dis 2009:36:331-5.

12 Pathela P, Braunstein SL, Schillinger JA, et al. Men who have sex with men have a 140-fold higher risk for newly diagnosed HIV and syphilis compared with heterosexual men in New York City. J Acquir Immune Defic Syndr 2011;58:408-16.

13 Jebbari $\mathrm{H}$, Simms I, Conti $\mathrm{S}$, et al. Variations in the epidemiology of primary, secondary and early latent syphilis, England and Wales: 1999 to 2008. Sex Transm Infect 2011:87:191-8.

14 Botham SJ, Ressler KA, Maywood P, et al. Men who have sex with men, infectious syphilis and HIV coinfection in inner Sydney: results of enhanced surveillance. Sex Health 2013;10:291-8.

15 Truong HM, Kellogg T, Klausner JD, et al. Increases in sexually transmitted infections and sexual risk behaviour without a concurrent increase in HIV incidence among men who have sex with men in San Francisco: a suggestion of HIV serosorting? Sex Transm Infect 2006:82:461-6.

16 Zetola NM, Klausner JD. Syphilis and HIV infection: an update. Clin Infect Dis 2007:44:1222-8

17 Marcus U, Bremer V, Hamouda O, et al. Understanding recent increases in the incidence of sexually transmitted infections in men having sex with men: changes in risk behavior from risk avoidance to risk reduction. Sex Transm Dis 2006;33:11-17.

18 Rompalo AM, Lawlor J, Seaman P, et al. Modification of syphilitic genital ulcer manifestations by coexistent HIV infection. Sex Transm Dis 2001;28:448-54.

19 Miller B, Hicks C. Syphilis and HIV: the intersection of two epidemics. J Watch HIV/ AIDS Clini Care. http://aids-clinical-care.jwatch.org/cgi/content/full/2010/903/1 (accessed Sep 2010).

20 Lynn WA, Lightman S. Syphilis and HIV: a dangerous combination. Lancet Infect Dis 2004;4:456-66

21 Sellati TJ, Wilkinson DA, Sheffield IS, et al. Virulent Treponema pallidum lipoprotein, and synthetic lipopeptides induce CCR5 on human monocytes and enhance their susceptibility to infection by human immunodeficiency virus type 1 . J Infect Dis 2000;181:283-93.

22 Buchacz K, Patel P, Taylor M, et al. Syphilis increases HIV viral load and decreases CD4 cell counts in HIV-infected patients with new syphilis infections. AIDS 2004;18:2075-9.

23 Palacios $\mathrm{R}$, Jiménez-Onate $\mathrm{F}$, Aguilar $\mathrm{M}$, et al. Impact of syphilis infection on HIV viral load and CD4 cell counts in HIV-infected patients. J Acquir Immune Defic Syndr 2007:44:356-9.

24 Rolfs RT, Joesoef MR, Hendershot EF, et al. A randomized trial of enhanced therapy for early syphilis in patients with and without human immunodeficiency virus infection. N Engl J Med 1997;337:307-14

25 Blank LJ, Rompalo AM, Erbelding EJ, et al. Treatment of syphilis in HIV-infected subjects: a systematic review of the literature. Sex Transm Infect 2011:87:9-16.

26 French $\mathrm{P}$, Gomberg $\mathrm{M}$, Janier $\mathrm{M}$, et al. IUSTI: 2008 European guidelines on the management of syphilis. Int J STD AIDS 2009;20:300-9. 


\section{Clinical}

27 Kingston $\mathrm{M}$, French $\mathrm{P}$, Goh $\mathrm{B}$, et al. UK national guidelines on the management of syphilis 2008. Int J STD AIDS 2008;19:729-40.

28 Workowski KA, Berman SM, et al. Sexually transmitted diseases treatment guidelines, 2010. Department of Health and Human Services, Centers for Disease Control and Prevention, 2010.

29 Hutchinson CM, Hook EW, Shepherd M, et al. Altered clinical presentation of early syphilis in patients with human immunodeficiency virus infection. Ann Intern Med 1994;121:94-9.

30 Hook EW, Behets F, Van Damme K, et al. A phase III equivalence trial of azithromycin versus benzathine penicillin for treatment of early syphilis. J Infect Dis 2010;201:1729-35.

31 Ghanem KG, Erbelding EJ, Cheng WW, et al. Doxycycline compared with benzathine penicillin for the treatment of early syphilis. Clin Infect Dis 2006;42: e45-9.
32 Wong $T$, Singh AE, De P. Primary syphilis: serological treatment response to doxycycline/tetracycline versus Benzathine Penicillin. Am J Med 2008;121: 903-8.

33 Long CM, Kalusner JD, Leon S, et al. Syphilis treatment and HIV infection in a population-based study of persons at high risk for sexually transmitted disease/HIV infection in Lima, Peru. Sex Transm Dis 2006;33:151-5.

34 Grimes M, Sahi SK, Godornes BC, et al. Two mutations associated with macrolide resistance in Treponema pallidum: increasing prevalence and correlation with molecular strain type in Seattle, Washington. Sex Transm Dis 2012;39: 954.

35 Tipple C, McClure MO, Taylor GP. High prevalence of macrolide resistant Treponema pallidum strains in a London centre. Sex Transm Infect 2011;87:486-8.

36 Lukehart SA, Godornes C, Molini BJ, et al. Macrolide resistance in Treponema pallidum in the United States and Ireland. N Eng/ J Med 2004;351:154-8. 